\title{
Ultrashort Long-Period Fiber Grating Sensors Inscribed on a Single Mode Fiber Using $\mathrm{CO}_{2}$ Laser Radiation
}

\author{
Marta Nespereira,' João M. P. Coelho,, ${ }^{1,2}$ Manuel Abreu, ${ }^{1}$ and José Manuel Rebordão ${ }^{1}$ \\ ${ }^{1}$ Laboratório de Óptica, Lasers e Sistemas, Faculdade de Ciências, Universidade de Lisboa, 1649-038 Lisboa, Portugal \\ ${ }^{2}$ Instituto de Biofísica e Engenharia Biomédica, Faculdade de Ciências, Universidade de Lisboa, 1749-016 Lisboa, Portugal \\ Correspondence should be addressed to Manuel Abreu; maabreu@fc.ul.pt
}

Received 30 May 2017; Revised 7 July 2017; Accepted 30 July 2017; Published 30 August 2017

Academic Editor: Stefania Campopiano

Copyright ( 2017 Marta Nespereira et al. This is an open access article distributed under the Creative Commons Attribution License, which permits unrestricted use, distribution, and reproduction in any medium, provided the original work is properly cited.

Sensing performances of ultrashort (as low as $2.4 \mathrm{~mm}$ ) long-period fiber gratings fabricated with $\mathrm{CO}_{2}$ laser radiation using commercial single mode fibers are presented. These lengths are, to our knowledge, the shortest of those found in the literature for this kind of sensors, approaching those typical in fiber Bragg gratings. Sensitivity to temperature and refractive index are demonstrated, with performances within the range expected for a single LPFG written on a single mode fiber without any enhancing technique. Analysis on results is made based on both theoretical and experimental data.

\section{Introduction}

Laser processing of optical fibers to produce fiber-based sensors has gained importance as a research theme, in particular regarding optical fiber grating sensors. These sensors are classified into two types: short-period gratings (also called reflection or fiber Bragg gratings, FBGs), in which coupling occurs between modes traveling in opposite directions, and transmission gratings (or long-period fiber gratings, LPFGs), where the coupling occurs between modes traveling in the same direction. Compared to other optical devices, LPFGs have a number of unique advantages: low insertion losses, high temperature sensitivity, and relatively simple fabrication. A further advantage of LPFG devices is their higher sensitivity to the environmental refractive index change without the need for access to the evanescent field, as in the case of the FBGs [1].

LPFGs have been developed and improved for decades, which resulted in numerous fabrication techniques and in a set of different sensor types, from simple LPFGs to interferometric systems, plasmonic resonance based sensing, and special fibers like photonic crystals [2-5]. There are many fabrication techniques inducing a permanent change on the refractive index: laser-based inscription systems such as UV [6], femtosecond pulses [7], and $\mathrm{CO}_{2}$ laser nearinfrared radiation [8], or physically deforming the fiber using other methods such as electric arc discharges [9-11], ion implantation [12], or microbends [13].

One important feature of LPFG systems is their potential to be endowed with different degrees of complexity, from single gratings to the application of different sensitivity enhancing techniques, such as mirrored ends [3], tapered LPFGs [14], or chemical etching [15]. Other techniques are based on SPR (Surface Plasmonic Resonance) configurations [5], different film coatings [16, 17], or nanospheres [18].

These gratings are fabricated with periods ranging from hundreds of micrometers to millimetres, and the majority have lengths of around $25-40 \mathrm{~mm}$; representative typical examples reported in the literature are summarized in Table 1 $[6,7,9,19-36]$. The two exceptions to this range (and the only ones we found in the literature) are the works of Wang [20] and Nam et al. [9]. The first engraves the LPFGs in PCF (Photonic Crystal Fiber) using a $\mathrm{CO}_{2}$ laser and the second uses electric arc for producing ultrashort LPFGs on regular single mode fibers.

In this paper, we present LPFGs written in single mode fibers (Corning SMF-28) using $\mathrm{CO}_{2}$ laser radiation $(10.6 \mu \mathrm{m}$ wavelength) with the remarkable characteristic of having a 
TABLE 1: Values of grating length, $L_{\text {grating }}$, found in the literature, for LPFGs written in regular SMF or PCF fibers. $\Lambda$ designates the grating period.

\begin{tabular}{|c|c|c|c|}
\hline Reference & Fabrication method & $L_{\text {grating }}(\mathrm{mm})$ & $\Lambda(\mu \mathrm{m})$ \\
\hline Slavík [19] & $\mathrm{CO}_{2}$ laser & 25-90 & 415 \\
\hline Wang [20] & $\mathrm{CO}_{2}$ laser & $2.8^{*}$ & $* *$ \\
\hline Rao and Zhu [21] & $\mathrm{CO}_{2}$ laser & 30 & $* *$ \\
\hline Vengsarkar et al. [22] & KrF laser & 25.4 & $* *$ \\
\hline Rao et al. [23] & $\mathrm{CO}_{2}$ laser & 40 & 2000 \\
\hline Lan et al. [24] & $\mathrm{CO}_{2}$ laser & 46 & $* *$ \\
\hline Oh et al. [25] & $\mathrm{CO}_{2}$ laser & $42,47,50$ & $* *$ \\
\hline Li et al. [26] & fs laser & 25.2 & 436 \\
\hline Han et al. [27] & UV light & 20 & 400 \\
\hline Zhang et al. [28] & Laser $^{* *}$ & 22.5 and 23 & $643,418,428$ \\
\hline Rindorf and Bang [29] & $\mathrm{CO}_{2}$ laser & $49.2,44.4,37.2,34.8^{*}$ & $820,740,620,580$ \\
\hline Allsop et al. [30] & $* *$ & 20 & 206 \\
\hline Allsop et al. [31] & $* *$ & 55,150 & 325,111 \\
\hline Shi et al. [32] & $* *$ & 13 & 435 \\
\hline Wang and Rao [33] & $\mathrm{CO}_{2}$ laser & 20 & $* *$ \\
\hline Rao et al. [34] & $\mathrm{CO}_{2}$ laser & 19.6 & 437 \\
\hline Wang and Rao [35] & $\mathrm{CO}_{2}$ laser & 27 & $* *$ \\
\hline Bhatia $[6,36]$ & UV & 10 & 280 \\
\hline Nam et al. [9] & Electric arc & 2 & 500 \\
\hline Duan et al. [7] & fs laser & 34 & 570 \\
\hline
\end{tabular}

*Engraved in a PCF (Photonic Crystal Fiber). ${ }^{* *}$ Not specified.

very short length (less than $10 \mathrm{~mm}$, in the range of $2.4 \mathrm{~mm}$ to $8.4 \mathrm{~mm}$ ). In comparison with the common 25-40 mm length LPFGs, these gratings are, to our knowledge, the shortest obtained so far using single mode fibers and $\mathrm{CO}_{2}$ laser radiation, very close to the typical lengths of FBGs (typically a few $\mathrm{mm}$ ). This makes them especially appealing for applications in which compactness is essential [37], or when the application requires probing small volumes. Using single mode fibers allows reducing the cost, when compared with using PCF, and using $\mathrm{CO}_{2}$ laser radiation allows better repeatability (compared with electric arc) and cheaper cost when compared with other laser technologies (like femtosecondbased technologies) and makes using photosensitive fibers unnecessary (required when using UV radiation). We also analyze the performance of the manufactured gratings as sensors of temperature and refractive index.

In the following section, we review the working principles of LPFGs, the physical bases of LPFGs as temperature and refractive index sensors, and a brief review of these two types of sensors in terms of their main characteristics. The experimental setup and methodologies will be explained in Section 3 and results will be presented and analyzed in Section 4 .

\section{Principles}

2.1. Working Principles of LPFG Sensors. As mentioned before, LPFGs are periodic structures engraved in the optical fiber along its longitudinal axes with the purpose of inducing a slight change in the refractive index of the fiber core.
They can be considered a particular case of FBGs, where the induced birefringence couples the light from the core (core mode) into the cladding, depending on the manufacturing technique and characteristics of the grating, such as period and length. The behavior of the different modes in a waveguide is described analytically by the coupled-mode theory [38]. Within the scope of this theory, the electric fields of the propagating modes are described by considering the grating as a dielectric perturbation that affects the effective refractive index of the propagation medium and the field amplitudes of the modes. The mathematical development requires calculating the effective refractive index of the medium for the core and cladding modes and the coupling constant, resulting in general coupled-mode equations [38]. The solution of these equations provides the transmission spectra of the grating, which relates the resonance wavelength, the grating's period, and the order of the coupled modes, expressed in the form $\mathrm{LP}_{l m}$ (acronym for "linearly polarized"). For symmetrical modes, $l=0$, with $m=1\left(\mathrm{LP}_{01}\right)$ for the fundamental mode. The asymmetric modes are referenced by $\mathrm{LP}_{l m}$, with $m=$ $1,2,3, \ldots$. The equation that summarizes the basic working principle of LPFGs is the phase matching condition [2]

$$
\lambda_{\text {res }}=\left[n_{\text {eff,core }}(\lambda)-n_{\text {eff,clad }}^{i}(\lambda)\right] \Lambda
$$

that relates the resonance wavelength $\lambda_{\text {res }}$ at which the core and a specific cladding mode are coupled, the difference between the effective refractive indexes of the core and the $i$ th cladding mode, $n_{\text {eff,core }}$ and $n_{\text {eff,clad }}^{i}$, respectively, and the grating's period $\Lambda$. 
LPFGs are characterized, among their fabrication parameters (period, length), by the coupling coefficient, $\kappa$, which describes the optimal coupling, according to

$$
\kappa L_{\text {grating }}=\frac{\pi}{2} \text {. }
$$

This expression shows that coupling depends directly on the grating's length, $L_{\text {grating, }}$, and the product $\kappa L_{\text {grating }}$ is known as "coupling strength" [37]. If $\kappa L_{\text {grating }}<\pi / 2$, the light is not completely coupled into a cladding mode, while if $\kappa L_{\text {grating }}>\pi / 2$, the light is first fully coupled into a cladding mode and then back-coupled again into a core mode, a phenomenon known as overcoupling [19]. The value of the coupling coefficient depends on the mechanical tension applied to the fiber and on the laser power, as both parameters affect the refractive index modulation in both core and cladding. In this kind of sensors, created under high tension, the coupling constant is usually high, in the order of $10 \mathrm{~cm}^{-1}$ [9]. Although $L_{\text {grating }}$ does not change the value of $\lambda_{\text {res }}$, the degree of coupling is affected, and the balance between $\kappa$ and $L_{\text {grating }}$ is usually established experimentally, while the LPFG is being created.

The sensitivity of a LPFG to an external parameter (induced or not) depends basically on the type of physical changes being induced in the grating. Temperature and refractive index are the examples that were considered in our work.

Grating sensitivity to temperature depends on two effects: one due to the change on the refractive indexes due to temperature variation and the other based on the thermomechanical sensitivity of the grating period [2].

By differentiation of the phase matching condition with respect to temperature, the sensitivity can be expressed mathematically by [2]

$$
\begin{aligned}
\frac{d \lambda_{\text {res }}}{d T}= & \frac{d \lambda_{0}}{d\left(\delta n_{\text {eff }}\right)}\left(\frac{d n_{\text {eff,core }}}{d T}-\frac{d n_{\mathrm{eff}_{\text {,clad }}}}{d T}\right) \\
& +\Lambda \frac{d \lambda_{\text {res }}}{d \Lambda} \frac{1}{L} \frac{d L}{d T} .
\end{aligned}
$$

In this equation, the first term of the second-hand side

$$
\frac{d \lambda_{0}}{d\left(\delta n_{\mathrm{eff}}\right)}\left(\frac{d n_{\mathrm{eff}, \text { core }}}{d T}-\frac{d n_{\mathrm{eff}_{\text {,clad }}{ }^{i}}}{d T}\right)
$$

represents the thermooptic effect (material contribution), where $\delta n_{\text {eff }}=\left(n_{\text {eff }}-n_{\text {clad }}\right)$. In addition, $\Lambda \cdot d \lambda_{\text {res }} / d \Lambda$ represents the contribution of the waveguide effect and $(1 / L) \cdot d L / d T$ is the thermal expansion coefficient.

The ability to use a LPFG to sense changes in the refractive index of the environment arises from the dependence of the effective refractive index for different $i$ th cladding modes upon the difference between the refractive index of the cladding and the refractive index of the surrounding medium; as shown in (1), the resonance depends on the effective refractive index of the aforesaid cladding modes
[2]. The variation of the transmitted wavelength with the refractive index is therefore given by [39]

$$
\frac{d \lambda}{d n_{\text {medium }}}=\frac{d \lambda}{d n_{\text {eff,clad }}^{i}} \frac{d n_{\text {eff,clad }}^{i}}{d n_{\text {medium }}} .
$$

The term $d n_{\text {eff,clad }}^{i} / d n_{\text {medium }}$ is different for different cladding modes; therefore, the sensitivity of the sensor depends on the specific mode being coupled to.

LPFGs created by $\mathrm{CO}_{2}$ laser radiation have some particularities regarding the three main physical mechanisms that modulate refractive index and mode coupling: residual stress relaxation, changes in the glass structure, and physical deformation. Residual stress relaxation is produced when LPFGs are written on fibers submitted to high tensile forces and results from different thermal expansion and viscoelastic properties between the core and the cladding. Changes in the glass structure are due to volume increase and glass densification, and the resulting physical deformations induce a variation of the effective refractive index along the fiber axis [40]. Since LPFGs are usually created by irradiating one side of the fiber, an asymmetrical refractive index profile is produced. This leads to an asymmetrical mode coupling, which can be considered a distinctive feature of $\mathrm{CO}_{2}$ laser induced LPFGs [20].

\subsection{LPFG-Based Sensors: Sensitivities to Temperature and} Refractive Index. In regard to sensing applications, LPGFs are based on well-known responses to different physical measurands, such as temperature, refractive index, torsion, bend, pressure, and strain [2, 21, 26-28, 33-35, 39, 41-44].

For temperature sensing, the typical values of sensitivities found in the literature range from around 0.05 to $0.3 \mathrm{~nm} /{ }^{\circ} \mathrm{C}$. The values are higher when more complex sensing structures were created, like, for example, a dual LPFG system [27]. Also, as mentioned by Nam et al. [9], sensitivity increases at high temperature: they measured a sensitivity of $0.054 \mathrm{~nm} /{ }^{\circ} \mathrm{C}$ from room temperature to $200^{\circ} \mathrm{C}$, while from $200^{\circ} \mathrm{C}$ to $1000^{\circ} \mathrm{C}$, the value was approximately $0.135 \mathrm{~nm} /{ }^{\circ} \mathrm{C}$ [9].

Regarding refraction index sensing, most authors report LPFGs' response to the refractive index in terms of resolution, publishing values one or two orders of magnitude higher than those measured by Abbe refractometers. The resolution values are around $10^{-4}$ to $10^{-6}$ RIU. As with temperature, such high values are obtained with more complex sensing systems instead of a single LPFG, such as the sandwiched system of three LPFGs in [27] or the Mach-Zehnder interferometer in [30]. A Photonic Crystal Fiber (PCF) was used by Rindorf and Bang [29].

\section{Experimental Setups and Methods}

The LPFGs fabrication technique consists in a focused $\mathrm{CO}_{2}$ laser beam (Synrad, $25 \mathrm{~W}$ ) periodically sweeping the fiber using an automatic system controlled by LabVIEW(, as described in detail in $[8,45]$. The average laser power was $4.5 \mathrm{~W}( \pm 1 \mathrm{~W})$, the focusing lens was cylindrical, with a working distance of $50 \mathrm{~mm}$, and the exposure time was $\sim 0.6 \mathrm{~s}$. 


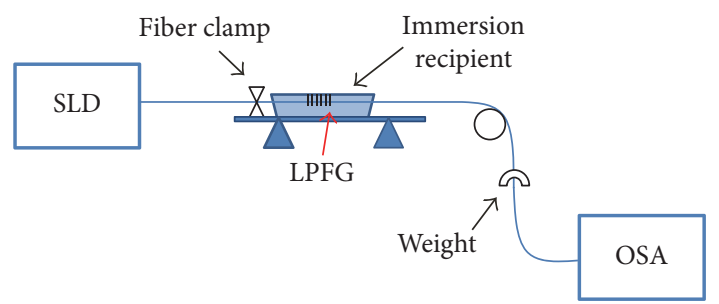

FIGURE 1: Schematic of the setup used for refractive index and temperature sensing tests.

A set of LPFGs were fabricated with a $600 \mu \mathrm{m}$ period and lengths varying between $2.4 \mathrm{~mm}$ and $4.8 \mathrm{~mm}$. A weight of around $80 \mathrm{~g}(0.78 \mathrm{~N})$ was applied to the engraving fibers, therefore inducing a high strain into the fibers. The period was chosen based on preliminary tests with our methodology [8]: it was one of those showing better repeatability and peak attenuation.

For temperature and refractive index measurements, the gratings were placed in a closed recipient filled with different fluids, as shown in Figure 1. Regarding temperature, the gratings were submerged in either boiling or frozen water, while a thermocouple was used for calibration. The corresponding spectra for each temperature were recorded in real time with an Optical Spectrum Analyzer (OSA) (Agilent 86140B) while the optical fiber was illuminated by a Super Luminescent Diode (SLD) with a central wavelength of $1550 \mathrm{~nm}$ and spectral width of $\sim 160 \mathrm{~nm}$. To test the sensors while measuring the refractive index, dissolution of ethylene glycol at different concentrations was used, having the refractive index of the solution previously measured with an Abbe refractometer (illuminated by a halogen lamp).

\section{Results and Analysis}

According to the coupled-mode theory (Section 2.1), and using a Matlab $\odot$ simulation [46-48], under the conditions previously described for the LPFGs, the coupling was found to occur between the $\mathrm{LP}_{01}$ core mode and the $\mathrm{LP}_{13}$ azimuthally asymmetric cladding mode $(m=3)$. This can be illustrated considering Figure 2, which shows the characteristic LPFG's phase matching curve, representing $\lambda_{\text {res }}$ versus $\Lambda$ for a grating with $6 \mathrm{~mm}$ length, and considering a 2-layer model and an asymmetrical mode [46-48]. The plot shows a set of periods $(\Lambda)$ complying with the phase matching condition (see (1)) for different resonance wavelengths. Each line represents a cladding mode, from the lowest-order mode $(m=1)$ to the highest-order mode $(m=7)$ (right to left). Then, for a period of $600 \mu \mathrm{m}$ and a resonance wavelength of around $1.5 \mu \mathrm{m}$, the mode has an order $m=3$.

For predicting the maximum refractive index changes induced in the core and cladding, we used a 3D Finite Element Model developed by the authors [8]. Since the experimental conditions are similar to those reported there, the behavior of the refractive index change of the core, $\Delta n_{\text {core }}$,

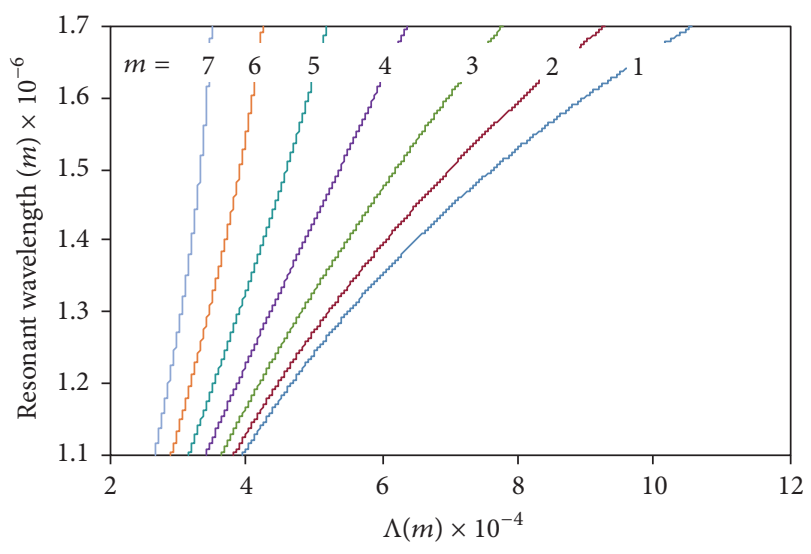

Figure 2: Phase matching curves, for a $6 \mathrm{~mm}$ length grating; $m$ indicates the order of the mode.

with the applied force, $F$, in Newton, can be considered linear and given by the empirical equation [8]

$$
\Delta n_{\text {core }}=-4.94 \times 10^{-4} \times F+1.66 \times 10^{-6} .
$$

The equivalent variation for the cladding was demonstrated to be two orders of magnitude smaller and not significantly changing with $F[8]$. Using the simulator mentioned before [46], it is then possible to study the variation in the attenuation signal at $\lambda_{\text {res }}$ for different applied weights or by changing the gratings length. Figure 3(a) exemplifies the change for a $4.8 \mathrm{~mm}$ length grating subjected to different weights. The plot shows that, for lower weights, the attenuation is small, which in practice limits the use of the grating as a sensor. Similarly, when using a low weight, in order to produce a useful sensing device, a grating with higher length is required. This effect is exemplified in Figure 3(b), representing the attenuation at $\lambda_{\text {res }}$ for different grating lengths, with a fixed weight of $5 \mathrm{~g}$ $(0.049 \mathrm{~N})$ when compared with the equivalent Figure 3(c) for a fixed weight of $80 \mathrm{~g}(0.78 \mathrm{~N})$.

The range assumed for the latter plot is reduced because although theoretically one can use higher weights when producing longer LPFGs (and obtain attenuations down to $-700 \mathrm{dBm}$ for a $50 \mathrm{~mm}$ LPFG), in practice this usually leads to fiber breakage, due to the combined effect of glass melting and weight pulling. Even when manufacturing shorter LPFGs with heavier weights, as we did, some tapering effect (and the resulting cladding thickness modulation) appears, as shown in Figure 4 (which shows a microscope photograph of an irradiated fiber under the experimental conditions considered in this work). The imaged zone comprises one $600 \mu \mathrm{m}$ period of a grating irradiated with $4.5 \mathrm{~W}$, for a duration of $0.6 \mathrm{~s}$ (each pulse), and subjected to a weight of $80 \mathrm{~g}(0.78 \mathrm{~N})$.

Thus, although higher pulling forces allow shorter length LPFGs, as predicted by theory, manufacturing can be affected by the resulting pulling force and the tapering effect can lead to breakage. Therefore, complete control of the process during the irradiation is mandatory. Taking this into consideration, with our experimental procedures, we were able to obtain LPFGs with significant attenuations at their resonance 


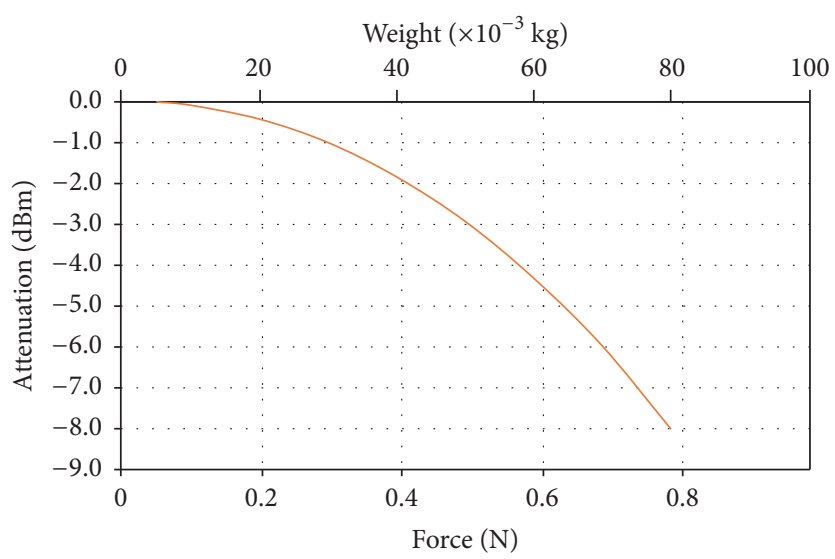

(a)

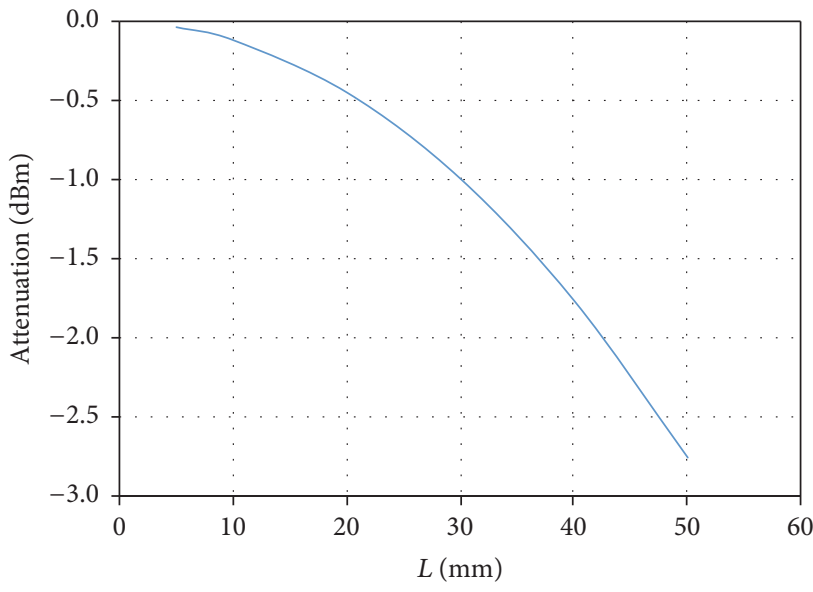

(b)

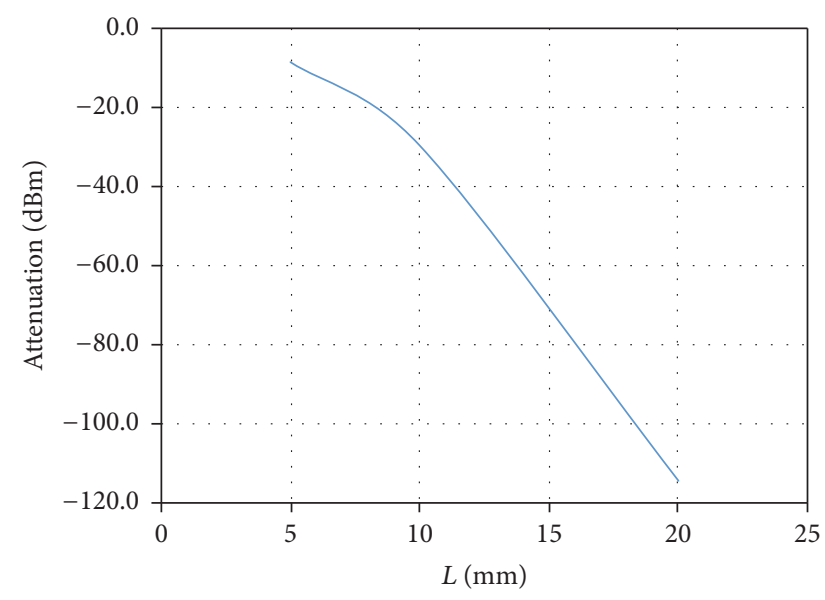

(c)

FIGURE 3: Theoretical attenuation at the resonance peak of a $600 \mu \mathrm{m}$ period LPFG when considering (a) a length of $4.8 \mathrm{~mm}$ and different weights and (b) $5 \mathrm{~g}(0.049 \mathrm{~N})$ and $(\mathrm{c}) 80 \mathrm{~g}(0.78 \mathrm{~N})$ weights and different length.

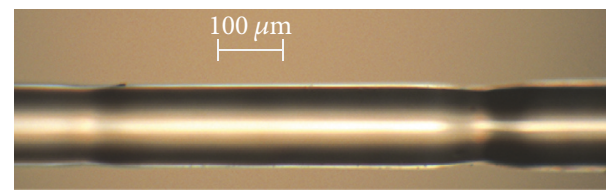

FIGURE 4: Micrograph showing the tapering effect in the irradiated zones in a $2.4 \mathrm{~mm}$ LPFG with $600 \mu \mathrm{m}$ period written on an SMF-28 optical fiber $\left(P \approx 4.5 \mathrm{~W} ; t_{\text {on }}=0.6 \mathrm{~s} ; F \approx 0.78 \mathrm{~N}\right)$.

wavelength, $\lambda_{\text {res }}$. Figure 5 exemplifies the spectra obtained for two ultrashort LPFGs. Their responses to variations of temperature and environment refractive index are analyzed hereafter. For each of the measurands, we will exemplify with the shorter length that still allows obtaining quality sensors (regarding repeatability and/or attenuation).

4.1. Temperature Sensing. Using the assembly sketched in Figure 1, it was experimentally observed that while the surrounding temperature increases, the resonance peak shifts towards higher wavelengths, while the amplitude (intensity) of the peak decreases, becoming less sharp. Figure 6 shows typical spectra, with the resonance moving towards shorter wavelengths while cooling down.

The response of the fiber is linear and sensitivities are within $0.06-0.08 \mathrm{~nm} /{ }^{\circ} \mathrm{C}$ in the temperature range of $25^{\circ} \mathrm{C}$ to $75^{\circ} \mathrm{C}$, for the LPFG lengths considered in our study. Figure 7 shows an example for a grating with a $600 \mu \mathrm{m}$ period and $4.8 \mathrm{~mm}$ length. The error in the linear adjustment obtained by the least squares method does not exceed $\pm 0.01 \mathrm{~nm} /{ }^{\circ} \mathrm{C}$. The resolution is obtained by dividing the resolution of the OSA (minimum wavelength at FWHM) by the sensitivity derived from the calibration line. In this case, the resolution ranges from $0.75^{\circ} \mathrm{C}$ to $1^{\circ} \mathrm{C}$.

In comparison, sensitivities are within the range of values presented in the literature for the temperature ranges considered in our study. Even considering higher ranges, although the values are not among the highest (e.g., [26, 27]), these gratings are much smaller than the regular ones, with a small number of "periods," and one of the factors affecting temperature sensitivity (wavelength effect) is directly connected to the number of periods, as discussed in Section 2.1. 


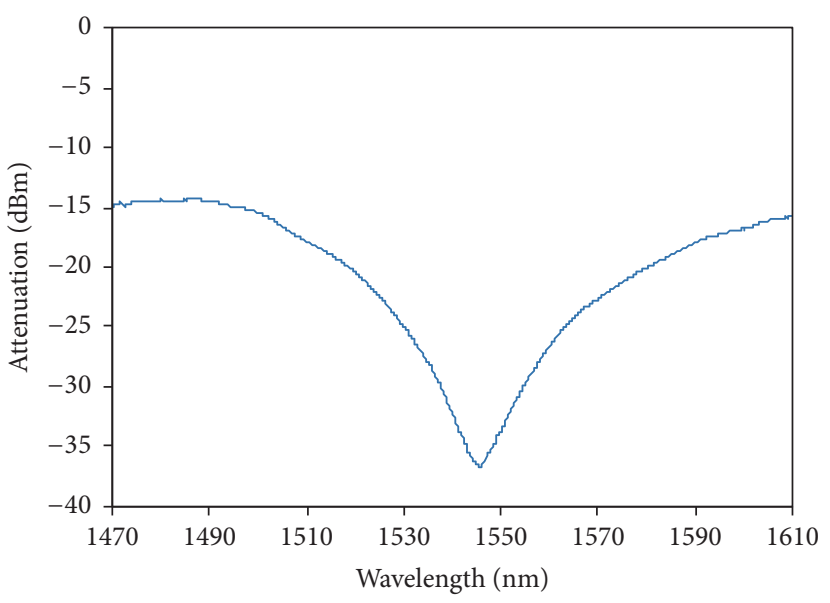

(a)

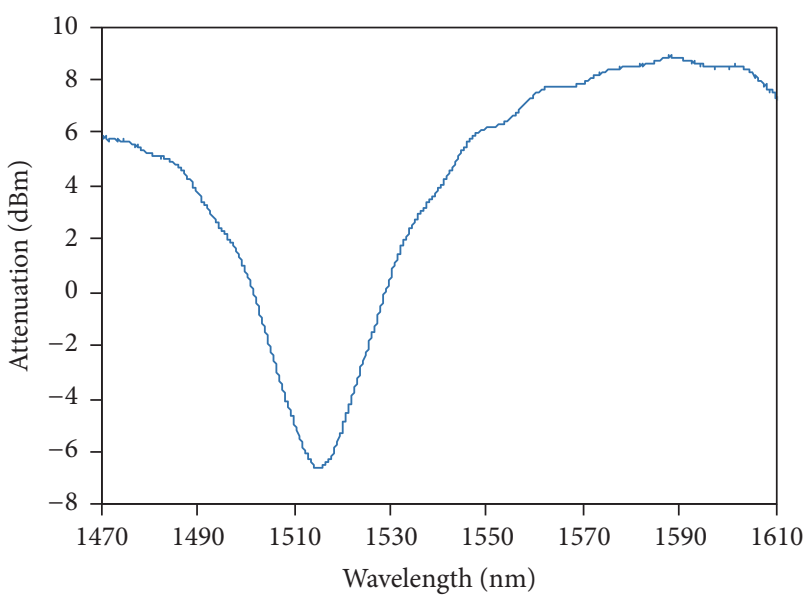

(b)

FIGURE 5: Transmission spectra (taken on air, at $22^{\circ} \mathrm{C}$ ) of two ultrashort LPFGs with (a) $2.4 \mathrm{~mm}$ and (b) $4.8 \mathrm{~mm} \mathrm{length,} \mathrm{a} 600 \mu \mathrm{m}$ period, written on SMF-28 optical fibers $\left(P \approx 4.5 \mathrm{~W} ; t_{\text {on }}=0.6 \mathrm{~s} ; F \approx 0.78 \mathrm{~N}\right)$.

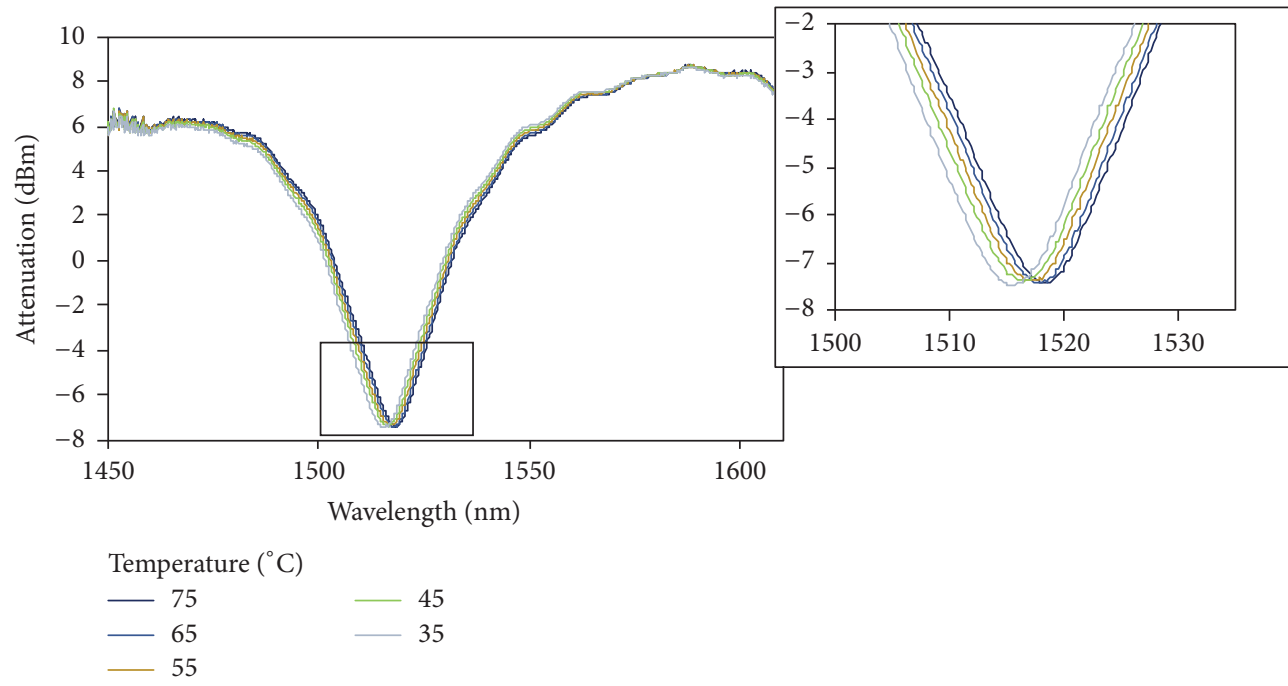

FIGURE 6: Example of the resonance shift with decreasing temperature of the medium for a $4.8 \mathrm{~mm}$ length grating.

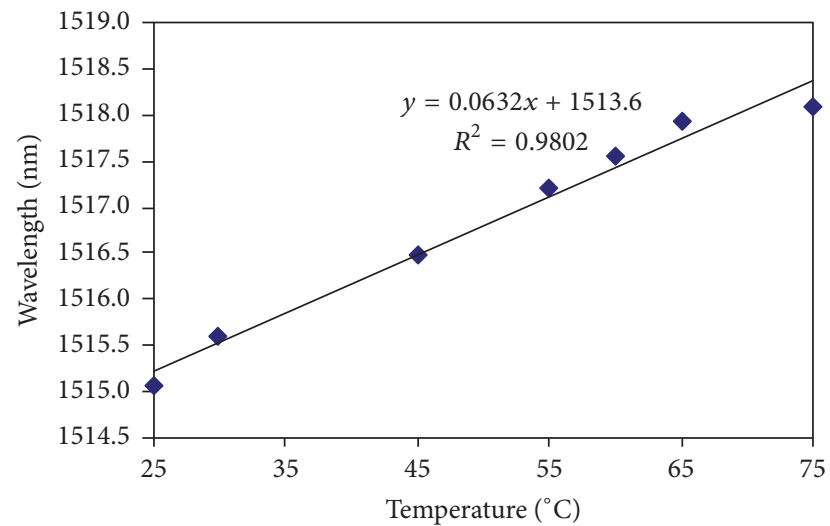

FIGURE 7: Variations with temperature of the resonance wavelength of a grating with $4.8 \mathrm{~mm}$ length.
4.2. Refractive Index Sensing. Using the same setup as for the temperature measurement testing (Figure 1), it was also observed that while increasing the refractive index of the surrounding environment, the resonance peak shifts linearly towards shorter wavelengths. Figure 8 shows a typical spectrum for a grating subjected to variations of the refractive index of the surrounding medium. In this case, the attenuation of the resonance peak becomes sharper and more pronounced while the refractive index increases. The increase of the attenuation could be explained by the absorption of this solvent at the infrared band, as explained in [49]. This potentially allows replacing an OSA by a much simpler power meter or by an Optical Time-Domain Reflectometer (OTDR), therefore leading to cheaper interrogation methods.

In Figure 9, we show some examples of the response of the gratings to changes of the refractive index, with an 


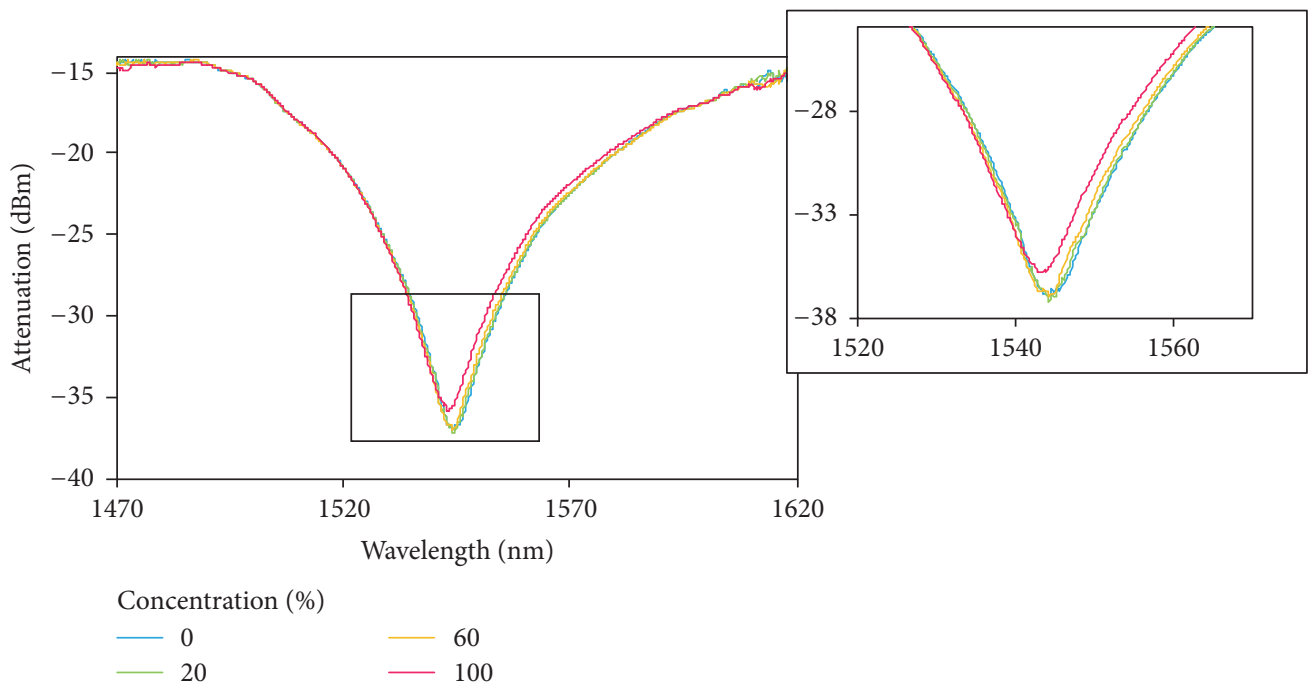

FIGURE 8: Example of the peak attenuation with the change in the refractive index of the medium (water with a variable percentage of ethylene glycol), for a grating with $2.4 \mathrm{~mm}$ length.

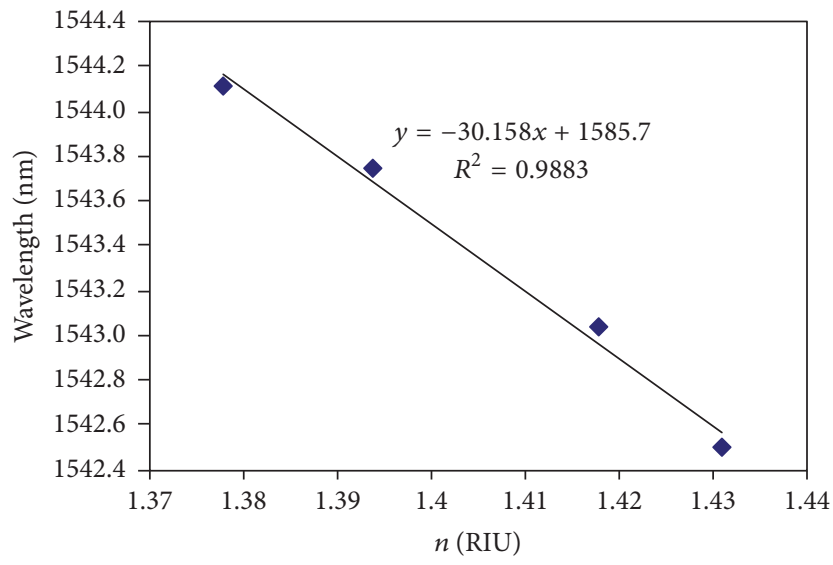

(a)

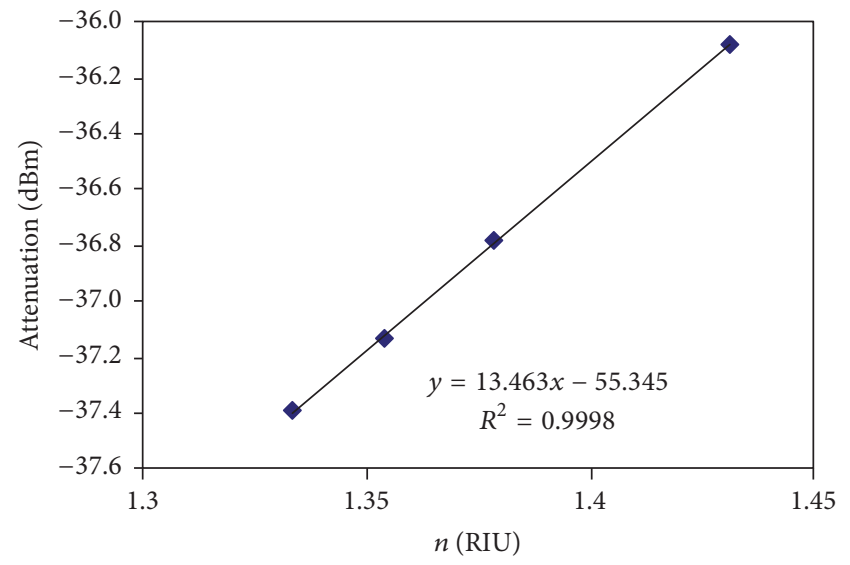

(b)

FIGURE 9: Responses of (a) the resonance wavelength and (b) attenuation of the signal with regard to the refractive index variation, for a grating with $2.4 \mathrm{~mm}$ length.

absolute sensitivity of around $30 \mathrm{~nm} / \mathrm{RIU}$ (about $14 \mathrm{~nm} / \mathrm{RIU}$, if we analyze the change in the attenuation of the signal), in the range of 1.333 to 1.431 RIU, which leads to resolutions in the order of magnitude of $10^{-3}$ RIU. The error in the linear adjustment is in the range of approximately $\pm 3 \mathrm{~nm} / \mathrm{RIU}$, obtained by the method of least squares.

The response of the gratings and their sensitivities are consistent with results reported for these types of sensors, although the comparison with the values mentioned in the literature (Section 2.2) shows that resolution is worse than that provided by a typical Abbe refractometer, which can be of the order of $10^{-5}$ RIU in the range of 1.33 to 1.58 RIU. In fact, the resolution of the OSA used in these experiments, $0.06 \mathrm{~nm}$, is actually limiting the resolution of these gratings.

\section{Conclusions}

The possibility of manufacturing ultrashort LPFGs by irradiating single mode fibers with $\mathrm{CO}_{2}$ laser radiation and high tension (up to $0.78 \mathrm{~N}$ ) was clearly demonstrated. Grating lengths (for a $600 \mu \mathrm{m}$ period) reached 2.4 and $4.8 \mathrm{~mm}$, far smaller than the 25 to $40 \mathrm{~mm}$ usually reported in the literature, but performances are alike. Performance of these LPFGs was assessed for measuring temperature and refractive index demonstrating,

(i) for the temperature, sensitivities in the interval $0.06-0.08 \mathrm{~nm} /{ }^{\circ} \mathrm{C}$ in the measuring range of 25 to $75^{\circ} \mathrm{C}$ and for the overall LPFG lengths being studied;

(ii) for the refractive index, sensitivities of around $-30 \mathrm{~nm} / \mathrm{RIU}$ in the measuring range of 1.333 to 1.431 RIU.

These values are fully consistent with the results reported in the literature for much longer LPFGs written in single mode fibers and under similar sensing operation, therefore demonstrating the sensing capabilities of our much shorter 
sensors. We anticipate that these small LPFGs demonstrate a high potential for applications requiring small sizes and compact sensing, especially in the areas of biosensing with less invasive devices, or civil engineering and structural health, by facilitating the embedding into the hosting material. Postinscription application of enhancing techniques like nanoparticles deposition is expected to give these gratings an even better sensitivity. Future work will follow this line of research and will explore the use of these ultrashort length gratings in the creation of more complex sensors, as concatenated LPFGs.

\section{Conflicts of Interest}

The authors declare that they have no conflicts of interest.

\section{Acknowledgments}

This work was partially supported through national funding by the Fundação para a Ciência e Tecnologia (FCT) through Project UID/BIO/00645/2013. The authors gratefully thank José Luis Santos, Orlando Frazão, Pedro Jorge, and Paulo Caldas from INESC-Porto for their advice and contributions. Special thanks are due to Catarina Silva, David Castro Alves, Fernando Monteiro, and António Oliveira for their support.

\section{References}

[1] J. M. P. Coelho, M. Nespereira, C. Silva, D. Pereira, and J. Rebordão, "Advances in optical fiber laser micromachining for sensors development," in Current Developments in Optical Fiber Technology, pp. 375-401, InTech, 2013.

[2] S. W. James and R. P. Tatam, "Optical fibre long-period grating sensors: characteristics and application," Measurement Science and Technology, vol. 14, no. 5, pp. R49-R61, 2003.

[3] P. L. Swart, "Long-period grating Michelson refractometric sensor," Measurement Science and Technology, vol. 15, no. 8, pp. 1576-1580, 2004.

[4] W. Shin, Y. L. Lee, T. J. Eom, B.-A. Yu, and Y.-C. Noh, "Temperature insensitive strain sensor based on long period fiber grating pair in photonic crystal fibers," in Proceedings of the 2009 14th OptoElectronics and Communications Conference, OECC 2009, chn, July 2009.

[5] Y.-J. He, Y.-L. Lo, and J.-F. Huang, "Optical-fiber surfaceplasmon-resonance sensor employing long-period fiber gratings in multiplexing," Journal of the Optical Society of America B: Optical Physics, vol. 23, no. 5, pp. 801-811, 2006.

[6] V. Bhatia, "Applications of long-period gratings to single and multi-parameter sensing," Optics Express, vol. 4, no. 11, pp. 457466, 1999.

[7] J. Duan, Z. Xie, C. Wang et al., "Torsion sensing characteristics of long period fiber gratings fabricated by femtosecond laser in optical fiber," Optics and Laser Technology, vol. 83, pp. 94-98, 2016.

[8] J. M. P. Coelho, C. Silva, M. Nespereira, M. Abreu, and J. M. Rebordão, "Writing of Long Period Fiber Gratings Using $\mathrm{CO}_{2}$ Laser Radiation," in Advances in Optical Fiber Technology: Fundamental Optical Phenomena and Applications, pp. 287-314, InTech, 2015.
[9] S. H. Nam, C. Zhan, J. Lee et al., "Bend-insensitive ultra short long-period gratings by the electric arc method and their applications to harsh environment sensing and communication," Optics Express, vol. 13, no. 3, pp. 731-737, 2005.

[10] G. Humbert and A. Malki, "Characterizations at very high temperature of electric arc-induced long-period fiber gratings," Optics Communications, vol. 208, no. 4-6, pp. 329-335, 2002.

[11] S. Kosinski and A. Vengsarkar, "Splicer-based long-period fiber gratings," in Proceedings of the OFC '98 Optical Fiber Communication Conference and Exhibit. Technical Digest Conference Edition 1998 OSA Technical Digest Series. Vol.2, pp. 278-279, San Jose, CA, USA.

[12] M. Fujimaki, Y. Ohki, J. L. Brebner, and S. Roorda, "Fabrication of long-period optical fiber gratings by use of ion implantation," Optics Letters, vol. 25, no. 2, pp. 88-89, 2000.

[13] I. K. Hwang, S. H. Yun, and B. Y. Kim, "Long-period fiber gratings based on periodic microbends," Optics Letters, vol. 24, no. 18, pp. 1263-1265, 1999.

[14] P.-H. Lu, K.-C. Hsu, S.-S. Jyu, and Y. Lai, "Periodically tapered long-period fiber gratings by $\mathrm{CO}_{2}$ laser heating and tension stretching," in Proceedings of the 15th Optoelectronics and Communications Conference (Oecc), pp. 626-627, 2010.

[15] K. Zhou, H. Liu, and X. Hu, "Tuning the resonant wavelength of long period fiber gratings by etching the fiber's cladding," Optics Communications, vol. 197, no. 4-6, pp. 295-299, 2001.

[16] Z. Wang, J. R. Heflin, K. Van Cott, R. H. Stolen, S. Ramachandran, and S. Ghalmi, "Biosensors employing ionic selfassembled multilayers adsorbed on long-period fiber gratings," Sensors and Actuators B: Chemical, vol. 139, no. 2, pp. 618-623, 2009.

[17] Z. Wang, J. R. Heflin, R. H. Stolen, and S. Ramachandran, "Analysis of optical response of long period fiber gratings to nmthick thin-film coatings," Optics Express, vol. 13, no. 8, pp. 28082813, 2005.

[18] D. Viegas, J. Goicoechea, J. M. Corres et al., "A fibre optic humidity sensor based on a long-period fibre grating coated with a thin film of $\mathrm{SiO}_{2}$ nanospheres," Measurement Science and Technology, vol. 20, no. 3, Article ID 034002, 2009.

[19] R. Slavík, "Extremely deep long-period fiber grating made with CO2 laser," IEEE Photonics Technology Letters, vol. 18, no. 16, pp. $1705-1707,2006$.

[20] Y. Wang, "Review of long period fiber gratings written by $\mathrm{CO} 2$ laser," Journal of Applied Physics, vol. 108, no. 8, Article ID 081101, 2010.

[21] Y. Rao and T. Zhu, "A novel flat-band long period grating with special apodization induced by high frequency $\mathrm{CO}_{2}$ laser pulses," in Proceedings of the the Optical Fiber Communication Conference, Optical Society of America, Anaheim, CA, USA, 2006.

[22] A. M. Vengsarkar, P. J. Lemaire, J. B. Judkins, V. Bhatia, T. Erdogan, and J. E. Sipe, "Long-period fiber gratings as bandrejection filters," Journal of Lightwave Technology, vol. 14, no. 1, pp. 58-64, 1996.

[23] Y. J. Rao, T. Zhu, and Q. J. Mo, "Highly sensitive fiber-optic torsion sensor based on an ultra-long-period fiber grating," Optics Communications, vol. 266, no. 1, pp. 187-190, 2006.

[24] X. Lan, Q. Han, T. Wei, J. Huang, and H. Xiao, “Turn-aroundpoint long-period fiber gratings fabricated by $\mathrm{CO} 2$ Laser pointby-point irradiations," IEEE Photonics Technology Letters, vol. 23, no. 22, pp. 1664-1666, 2011. 
[25] S. T. Oh, W. T. Han, U. C. Paek, and Y. Chung, "Azimuthally symmetric long-period fiber gratings fabricated with $\mathrm{CO} 2$ laser," Microwave and Optical Technology Letters, vol. 41, no. 3, pp. 188-190, 2004.

[26] B. Li, L. Jiang, S. Wang, H.-L. Tsai, and H. Xiao, "Femtosecond laser fabrication of long period fiber gratings and applications in refractive index sensing," Optics and Laser Technology, vol. 43, no. 8, pp. 1420-1423, 2011.

[27] Y.-G. Han, S. B. Lee, C.-S. Kim, J. U. Kang, U.-C. Faek, and Y. Chung, "Simultaneous measurement of temperature and strain using dual long-period fiber gratings with controlled temperature and strain sensitivities," Optics Express, vol. 11, no. 5, pp. 476-481, 2003.

[28] A. P. Zhang, L.-Y. Shao, J.-F. Ding, and S. He, "Sandwiched longperiod gratings for simultaneous measurement of refractive index and temperature," IEEE Photonics Technology Letters, vol. 17, no. 11, pp. 2397-2399, 2005.

[29] L. Rindorf and O. Bang, "Highly sensitive refractometer with a photonic-crystal-fiber long-period grating," Optics Letters, vol. 33, no. 6, pp. 563-565, 2008.

[30] T. Allsop, R. Reeves, D. J. Webb, I. Bennion, and R. Neal, "A high sensitivity refractometer based upon a long period grating Mach-Zehnder interferometer," Review of Scientific Instruments, vol. 73, no. 4, p. 1702, 2002.

[31] T. Allsop, L. Zhang, and I. Bennion, "Detection of organic aromatic compounds in paraffin by a long-period fiber grating optical sensor with optimized sensitivity," Optics Communications, vol. 191, no. 3-6, pp. 181-190, 2001.

[32] L. Shi, T. Zhu, Y.-E. Fan, K. S. Chiang, and Y. Rao, "Torsion sensing with a fiber ring laser incorporating a pair of rotary long-period fiber gratings," Optics Communications, vol. 284, no. 22, pp. 5299-5302, 2011.

[33] Y. P. Wang and Y. J. Rao, "Long period fibre grating torsion sensor measuring twist rate and determining twist direction simultaneously," Electronics Letters, vol. 40, no. 3, pp. 164-166, 2004.

[34] Y.-J. Rao, Y.-P. Wang, Z.-L. Ran, and T. Zhu, "Novel fiber-optic sensors based on long-period fiber gratings written by highfrequency $\mathrm{CO}_{2}$ laser pulses," Journal of Lightwave Technology, vol. 21, no. 5, pp. 1320-1327, 2003.

[35] Y.-P. Wang and Y.-J. Rao, "CO2-laser induced LPFG torsion characteristics depending on length of twisted fibre," Electronics Letters, vol. 40, no. 18, pp. 1101-1103, 2004.

[36] V. Bhatia, Properties and sensing applications of long-period gratings Ph.D. Dissertation [Ph.D. thesis], Virginia Tech, Blacksburg, Virginia, 1996.

[37] R. Haslinger, P. Leyendecker, and U. Seibold, "A fiberoptic force-torque-sensor for minimally invasive robotic surgery," in Proceedings of the 2013 IEEE International Conference on Robotics and Automation, ICRA 2013, pp. 4390-4395, deu, May 2013.

[38] P. Caldas, Fiber Optic Sensing by Evanescent Field Interaction [Ph.D. thesis], Department of Physics and Astronomy of the Faculty of Sciences of the University of Porto, 2011.

[39] J. H. Chong, P. Shum, H. Haryono et al., "Measurements of refractive index sensitivity using long-period grating refractometer," Optics Communications, vol. 229, no. 1-6, pp. 65-69, 2004.

[40] Y.-Q. Liu, W.-T. Tu, D. Yang, and T.-Y. Wang, "Fabrication of long-period fiber gratings by $\mathrm{CO} 2$ laser in fiber under tension," Journal of Shanghai University, vol. 15, no. 1, pp. 1-6, 2011.
[41] L. Qi, C.-L. Zhao, J. Yuan et al., "Highly reflective long period fiber grating sensor and its application in refractive index sensing," Sensors and Actuators, B: Chemical, vol. 193, pp. 185189, 2014

[42] I. Peshko, O. Cherry, T. Rutkevich, B. Hockley, and V. Rubtsov, "Long-period gratings in optical fibres for chemical sensor applications," Measurement Science and Technology, vol. 16, no. 11, pp. 2221-2228, 2005.

[43] Nidhi, R. S. Kaler, U. Tiwari, V. Mishra, N. Singh, and P. Kapur, "Investigation of long period grating as refractive index sensor," Optik, vol. 123, no. 12, pp. 1071-1073, 2012.

[44] R. Falciai, A. G. Mignani, and A. Vannini, "Long period gratings as solution concentration sensors," Sensors and Actuators, B: Chemical, vol. 74, no. 1-3, pp. 74-77, 2001.

[45] D. Castro Alves, J. M. P. Coelho, M. Nespereira, F. Monteiro, M. Abreu, and J. M. Rebordão, "Automation methodology for the development of LPFG using CO2 laser radiation," in Proceedings of the 8th Iberoamerican Optics Meeting, RIAO 2013 and 11th Latin American Meeting on Optics, Lasers, and Applications, OPTILAS 2013, prt, July 2013.

[46] F. D. V. Baptista, Simulação do comportamento espectral de redes de período longo em fibra óptica [M.Sc. Thesis], Centro de Ciências Exactas e da Engenharia, University of Madeira, Funchal, Portugal, 2009.

[47] D. Gloge, "Weakly guiding fibers," Applied Optics, vol. 10, no. 10, pp. 2252-2258, 1971.

[48] D. Gloge, “Dispersion in Weakly Guiding Fibers," Applied Optics, vol. 10, no. 11, pp. 2442-2445, 1971.

[49] X. Daxhelet and M. Kulishov, "Theory and practice of longperiod gratings: when a loss becomes a gain," Optics Letters, vol. 28 , no. 9 , pp. $686-688,2003$. 


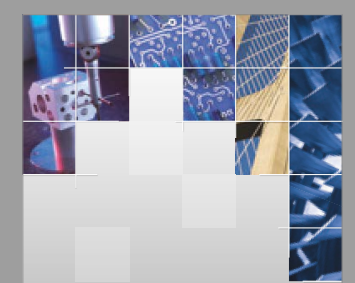

\section{Enfincering}
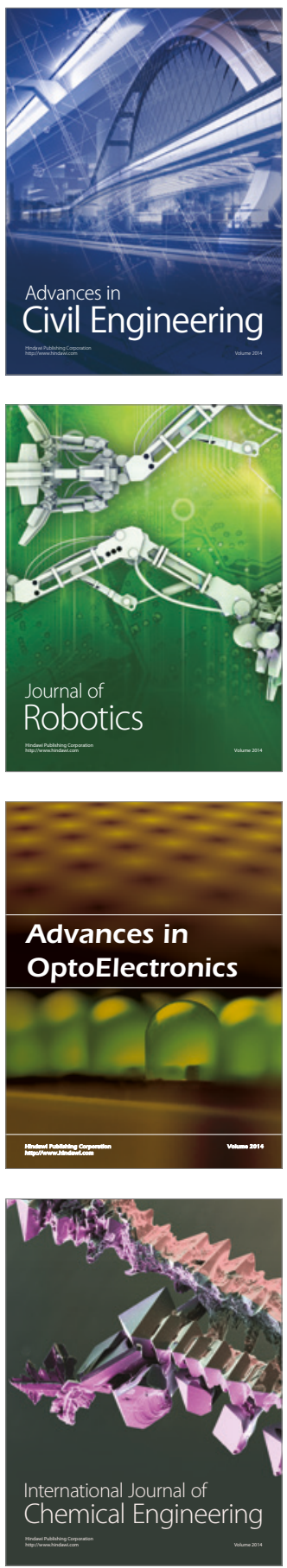

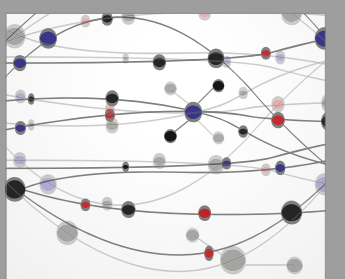

The Scientific World Journal

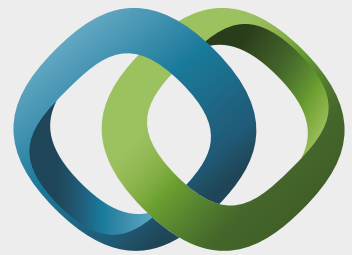

\section{Hindawi}

Submit your manuscripts at

https://www.hindawi.com
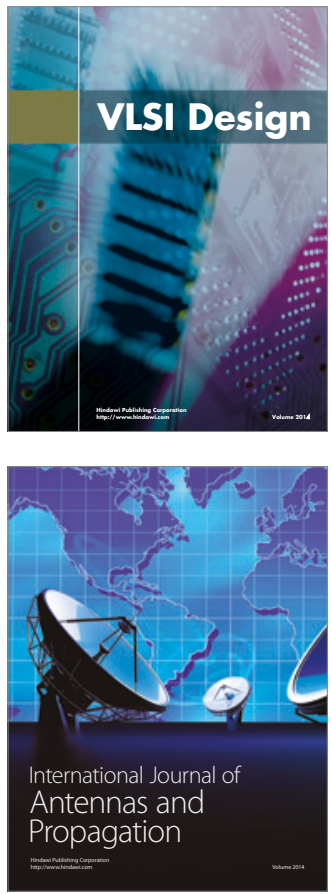

\section{Rotating}

Machinery
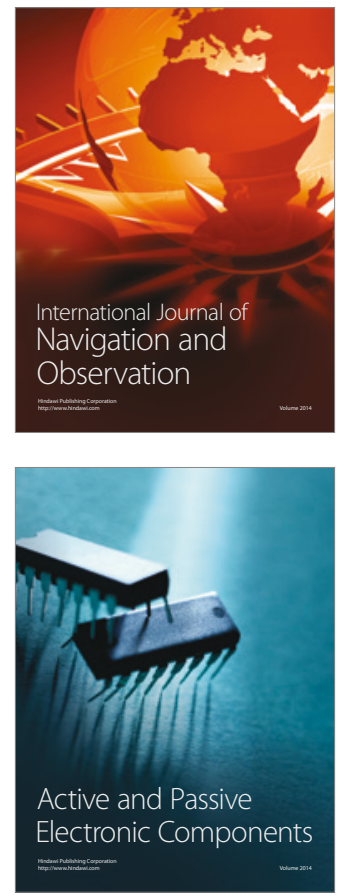
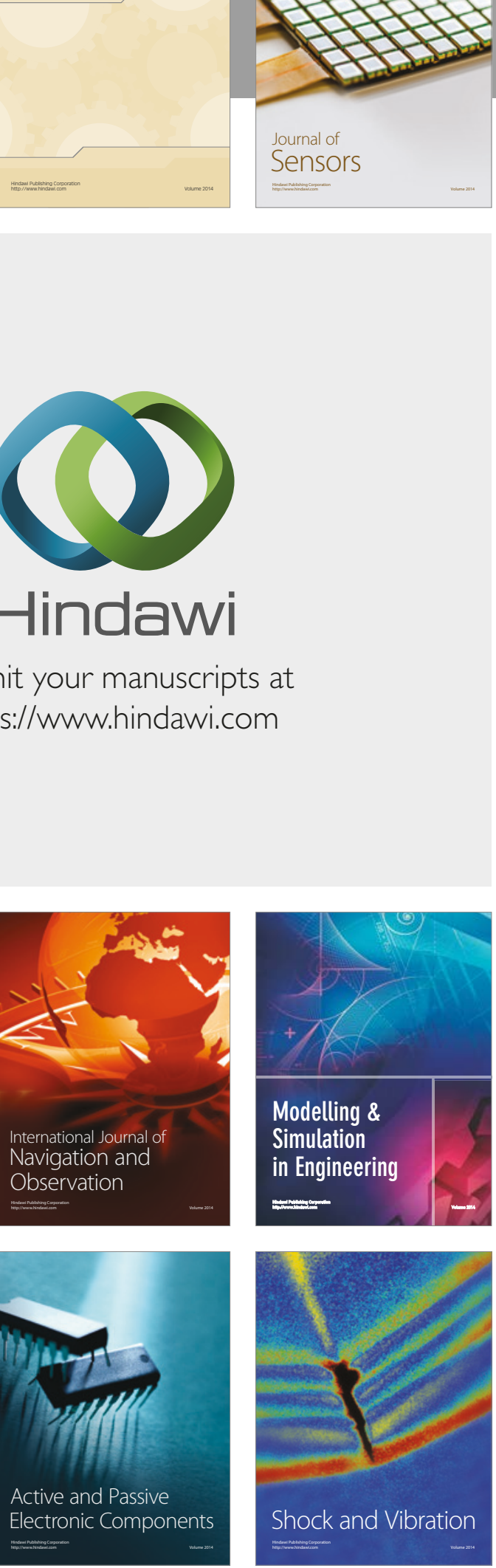
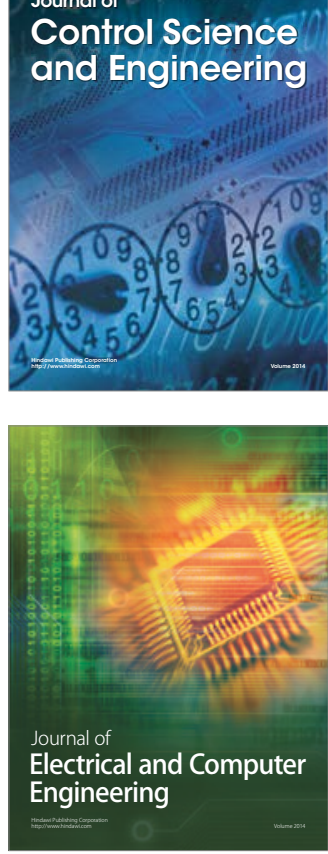

Distributed

Journal of

Control Science

and Engineering
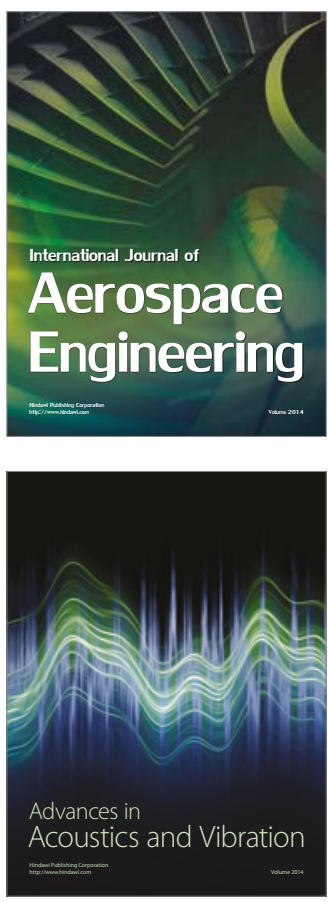

Sensor Networks 